\title{
Asiatic acid, a pentacyclic triterpene in Centella asiatica, attenuates glutamate-induced cognitive deficits in mice and apoptosis in SH-SY5Y cells
}

\author{
Min-fang XU1 ${ }^{1}$, Yu-yun XIONG ${ }^{2}$, Jian-kang LIU ${ }^{3}$, Jin-jun QIAN ${ }^{4}$, Li ZHU ${ }^{5}$, Jing GAO ${ }^{1, *}$ \\ ${ }^{1}$ School of Pharmacy, Jiangsu University, Zhenjiang 212013, China; ${ }^{2}$ School of Medical Science and Laboratory Medicine, Jiangsu \\ University, Zhenjiang 212013, China; ${ }^{3}$ Institute of Mitochondrial Biology \& Medicine, The Key Laboratory of Biomedical Information \\ Engineering of Ministry of Education, Xi'an Jiaotong University School of Life Science and Technology, Xi'an 710049, China; ${ }^{4}$ Depart- \\ ment of Neurology, The Forth Affiliated Hospital of Jiangsu University, Zhenjiang 212001, China; ${ }^{5}$ Department of Neurobiology and \\ Neurochemistry, Institute for Nautical Medicine and Jiangsu Key Laboratory of Neurogeneration, Nantong University, Nantong 226001, \\ China
}

Aim: To investigate whether asiatic acid (AA), a pentacyclic triterpene in Centella asiatica, exerted neuroprotective effects in vitro and in vivo, and to determine the underlying mechanisms.

Methods: Human neuroblastoma SH-SY5Y cells were used for in vitro study. Cell viability was determined with the MTT assay. Hoechst 33342 staining and flow cytometry were used to examine the apoptosis. The mitochondrial membrane potential (MMP) and reactive oxygen species (ROS) were measured using fluorescent dye. PGC-1 $\alpha$ and Sirt1 levels were examined using Western blotting. Neonatal mice were given monosodium glutamate $(2.5 \mathrm{mg} / \mathrm{g})$ subcutaneously at the neck from postnatal day (PD) 7 to 13 , and orally administered with AA on PD 14 daily for $30 \mathrm{~d}$. The learning and memory of the mice were evaluated with the Morris water maze test. HE staining was used to analyze the pyramidal layer structure in the CA1 and CA3 regions.

Results: Pretreatment of SH-SY5Y cells with AA (0.1-100 nmol/L) attenuated toxicity induced by 10 mmol/L glutamate in a concentration-dependent manner. AA $10 \mathrm{nmol} / \mathrm{L}$ significantly decreased apoptotic cell death and reduced reactive oxygen species (ROS), stabilized the mitochondrial membrane potential (MMP), and promoted the expression of PGC-1 $\alpha$ and Sirt1. In the mice models, oral administration of AA (100 mg/kg) significantly attenuated cognitive deficits in the Morris water maze test, and restored lipid peroxidation and glutathione and the activity of SOD in the hippocampus and cortex to the control levels. AA (50 and $100 \mathrm{mg} / \mathrm{kg}$ ) also attenuated neuronal damage of the pyramidal layer in the CA1 and CA3 regions.

Conclusion: AA attenuates glutamate-induced cognitive deficits of mice and protects SH-SY5Y cells against glutamate-induced apoptosis in vitro.

Keywords: asiatic acid; glutamate; excitotoxicity; cognitive deficits; hippocampus; apoptosis; mitochondria; PGC-1 $\alpha$; neuroprotection

Acta Pharmacologica Sinica (2012) 33: 578-587; doi: 10.1038/aps.2012.3; published online 26 Mar 2012

\section{Introduction}

Human neurodegenerative diseases, including Alzheimer's disease, Parkinson's disease, and amyotrophic lateral sclerosis, are characterized by the progressive dysfunction and loss of neurons induced by particular neurological deficits ${ }^{[1]}$. Glutamate (Glu)-induced excitotoxicity plays an important role in the pathogenesis of these diseases ${ }^{[2,3]}$. Glu-induced neuronal death is initiated by overstimulation of $N$-methyl- $D$-aspartate (NMDA) receptors, resulting in an increase in intracellular free

\footnotetext{
* To whom correspondence should be addressed.

E-mail jinggao@ujs.edu.cn

Received 2011-09-13 Accepted 2012-01-10
}

calcium, followed by the activation of catabolic enzymes and leading to an intracellular cascade of cytotoxic events.

Recently, an increasing number of studies has found that mitochondria - organelles that are vitally important for controlling cell life and death - are involved in Glu-induced excitotoxicity because they possess a large capacity for calcium uptake in response, finally resulting in mitochondrial $\mathrm{Ca}^{2+}$ overload. Mitochondrial $\mathrm{Ca}^{2+}$ overload may activate neuronal cell death through the release of pro-apoptotic factors and increased generation of reactive oxygen species $(\mathrm{ROS})^{[4,5]}$. The extent to which ROS and subsequent oxidative stress may play an essential role in Glu toxicity in both acute insults, such as ischemia ${ }^{[6]}$, and chronic neurodegenerative diseases ${ }^{[7]}$ has been 
investigated. Thus, oxidative damage, destruction of calcium homeostasis and mitochondrial dysfunction are essentially consequences of Glu-induced excitotoxicity. Consistent with these findings, antioxidants and mitochondrial nutrients ${ }^{[8,9]}$ may be promising candidates for the prevention and treatment of these diseases.

Centella asiatica has long been used in Ayurvedic medicine and traditional Chinese medicine to treat various ailments and to enhance memory. Recent findings suggest that Centella asiatica has cognition-enhancing properties through its ability to protect against oxidative stress ${ }^{[10,11]}$, reduce the extent of mitochondrial damage $\mathrm{e}^{[12]}$ and increase axonal regeneration and neurite elongation ${ }^{[13]}$. Asiatic acid (AA) is a pentacyclic triterpene found in Centella asiatica. Our previous studies demonstrated that AA could attenuate $\mathrm{H}_{2} \mathrm{O}_{2}$ - or rotenone-induced neural injury due to its protection from mitochondrial membrane depolarization ${ }^{[14]}$. AA also showed protective effects against Glu- and $A \beta$-induced neurotoxicity ${ }^{[15,16]}$. Moreover, AA may be an effective agent for treating cerebral ischemia ${ }^{[17]}$. Therefore, AA is interesting as a candidate for potential application in the treatment of neurodegenerative diseases.

In this study, we used an in vitro model of Glu-induced excitotoxicity in SH-SY5Y cells and an in vivo dementia model of perinatal monosodium glutamate (MSG) exposure ${ }^{[18]}$ to investigate the neuroprotective functions of $\mathrm{AA}$ and its possible mechanisms of action.

\section{Materials and methods Materials}

Glu, MSG, AA and MTT were purchased from Sigma (St Louis, MO, USA). Minimum Essential Medium (MEM), Nutrient Mixture Ham's F-12 (F12), nonessential amino acids and trypsin were purchased from Gibco BRL (Grand Island, NY, USA). Fetal bovine serum (FBS) was obtained from Sijiqing Biological Engineering Materials (Hangzhou, China). DCFHDA and the BCA Protein Quantitative Analysis Kit were purchased from Beyotime (Nantong, China). Anti- $\beta$-actin primary antibody was purchased from Abcam (Cambridge, MA, USA). Anti-PGC-1 $a$ and anti-Sirt1 antibodies were purchased from Santa Cruz Biotechnology (San Diego, CA, USA), and all secondary antibodies were purchased from Boster Biological Technology (Wuhan, China). All other reagents were purchased from commercial suppliers and were of standard biochemical quality.

\section{Culture of SH-SY5Y Cells}

Human neuroblastoma SH-SY5Y cells (a gift from Dr Zun-ji KE, Institute for Nutritional Sciences, Chinese Academy of Sciences, Shanghai, China) were maintained in MEM/F12 medium, supplemented with $1 \%$ nonessential amino acids and $10 \% \mathrm{FBS}, 100 \mathrm{U} / \mathrm{mL}$ penicillin and $100 \mathrm{U} / \mathrm{mL}$ streptomycin at $37^{\circ} \mathrm{C}$ in $5 \% \mathrm{CO}_{2}$. The cells were passaged once every $3 \mathrm{~d}$.

\section{MTT assay}

To determine cell viability, the MTT assay was used. SHSY5Y cells were cultured in 96-well plates at a seeding den- sity of 3000 cells per well. Twenty-four hours later, the cells were treated with AA for $24 \mathrm{~h}$ and then exposed to the same fresh medium containing $10 \mathrm{mmol} / \mathrm{L}$ Glu for $24 \mathrm{~h}$. Next, $100 \mu \mathrm{L} /$ well MTT $(1 \mathrm{mg} / \mathrm{mL})$ was added to each well, and the cells were incubated for $4 \mathrm{~h}$ at $37^{\circ} \mathrm{C}$. After incubation, dimethyl sulfoxide (DMSO, $100 \mu \mathrm{L}$ ) was added to each well to dissolve the precipitate. The absorbance was read with a microplate reader (Molecular Devices, Sunnyvale, CA, USA) at $570 \mathrm{~nm}$.

\section{Flow cytometric analysis}

Briefly, following drug treatment, the cells were harvested and washed twice with ice-cold PBS. The presence of apoptotic cells that expose phosphatidylserine on their outside surface was determined by an Annexin V-FITC Apoptosis Kit (Calbiochem, San Diego, CA, USA). Annexin V-FITC (1.25 $\mu \mathrm{L})$ was added to $500 \mu \mathrm{L}$ of $1 \times$ Annexin V-FITC binding buffer, and the cells were incubated at room temperature for $15 \mathrm{~min}$ in the dark. After washing the cells with $1 \times$ binding buffer, $10 \mu \mathrm{L}$ propidium iodide (PI) was added to the binding buffer, and the cells were analyzed with a flow cytometer (BeckmanCoulter MoFLo XDP, Fullerton, CA, USA). The percentages of apoptotic and necrotic cells were estimated for each sample.

\section{Mitochondrial membrane potential (MMP) assay}

The MMP were determined with a fluorescent dye, JC-1 (Molecular Probes, Eugene, OR, USA). The cells were seeded in $24-w e l l$ plates at a density of $3 \times 10^{4}$ cells $/ \mathrm{mL}$. The fluorescence intensity was observed immediately following JC-1 staining $\left(2.5 \mathrm{\mu g} / \mathrm{mL}\right.$ of JC- 1 at $37^{\circ} \mathrm{C}$ for $\left.30 \mathrm{~min}\right)$ with fluorescence spectrometry (Molecular Devices, Sunnyvale, CA, USA; Ex 488/Em 535 for JC-1 green and Ex 488/Em 595 for JC-1 red) and fluorescence microscopy (Nikon TE2000 inverted microscope, Tokyo, Japan).

\section{Hoechst 33342 staining}

The cells were cultured in 24-well plates at a density of $3 \times 10^{4}$ cells $/ \mathrm{mL}$. The cells were fixed in $4 \%$ paraformaldehyde for $30 \mathrm{~min}$ at room temperature. After staining with $10 \mu \mathrm{g} / \mathrm{mL}$ Hoechst 33342 for $10 \mathrm{~min}$, the cells were observed under a fluorescence microscope.

\section{Intracellular ROS determination}

ROS were measured with the non-fluorescent probe DCFHDA. The cells were incubated with DCFH-DA at $37^{\circ} \mathrm{C}$ for $30 \mathrm{~min}$, and the distribution of DCF fluorescence produced by $1 \times 10^{4}$ cells was detected with a fluorescence microscope or FACscan cytometer at an excitation wavelength of $488 \mathrm{~nm}$ and an emission wavelength of $535 \mathrm{~nm}$.

\section{Western blot analysis}

After treatment like describing in MTT assay, $1 \times 10^{6}$ cells were collected and subjected to Western blot analysis. The cell proteins were extracted and quantified with a BCA Protein Quantitative Analysis Kit. After addition of the sample loading buffer, protein samples were electrophoresed using $8 \%-12 \%$ 
SDS-PAGE and subsequently transferred to PVDF membranes (Bio-Rad, Hercules, CA, USA). Each membrane was incubated in fresh blocking buffer $(0.1 \%$ Tween 20 in Tris-buffered saline, $\mathrm{pH} 7.4$, containing $5 \%$ nonfat dried milk) at room temperature for $30 \mathrm{~min}$ and then probed with anti-PGC-1a, anti-Sirt1 or anti- $\beta$-actin antibodies in blocking buffer at $4{ }^{\circ} \mathrm{C}$ overnight. The membrane was washed three times for 5 min each using PBST (PBS and 0.1\% Tween 20). The membrane was incubated in the appropriate HRP-conjugated secondary antibody at room temperature for $2 \mathrm{~h}$. The immunoreactive protein was visualized using the chemiluminescent reagent ECL (Pierce Biotechnology, Rockford, IL, USA) according to the manufacturer's protocol.

\section{Animals}

Neonatal mice at postnatal day (PD) 7 were procured from the Comparative Medicine Center (Yangzhou University, Yangzhou, China) and housed in cages at an ambient temperature of $25^{\circ} \mathrm{C}$ with $12 \mathrm{~h}$ light/dark cycles. Food and water were freely available.

\section{Drug administration}

The animals were randomly assigned to drug or control groups ( $n=10$ in each group), respectively. MSG was dissolved in $0.9 \%$ sodium chloride $(\mathrm{NaCl})$. In the drug group, neonatal mice were given MSG subcutaneously (in the neck, $2.5 \mathrm{mg} / \mathrm{g}$ body weight) from PD 7 to 13 as previously described ${ }^{[19]}$. The control pups received equal volumes of $0.9 \% \mathrm{NaCl}$. On PD 28 , the animals were weaned, and animals of the same sex that had been subjected to the same treatment were housed together. AA was suspended in $0.5 \%$ carboxymethylcellulose and administered by oral gavage. The animals were divided into four experimental groups: control, MSG, MSG $+50 \mathrm{mg} / \mathrm{kg}$ AA and MSG+100 mg/kg AA. The AA doses used in this study were chosen on the basis of previously published experiments $^{[17]}$. AA was administered after MSG treatment on PD 14 and daily thereafter for $30 \mathrm{~d}$. The mice then performed the Morris water maze. All protocols described were reviewed and approved.

\section{Morris water maze test}

The animals were tested with a spatial version of the Morris water maze $\mathrm{e}^{[20]}$. It consisted of a circular water tank $(90 \mathrm{~cm}$ diameter, $50 \mathrm{~cm}$ height) that was partially filled with water $\left(25 \pm 2{ }^{\circ} \mathrm{C}\right)$. Black ink was used to render the water opaque. Prior to the water maze testing, all mice were habituated to the water by being allowed to swim freely without a platform present. The pool was in the center of a room containing various salient visual cues and was divided virtually into four equal quadrants, labeled N (north), S (south), E (east), and W (west). The cues remained constant throughout the testing process. An escape platform (6 cm diameter) was hidden 1 $\mathrm{cm}$ below the water surface in one of the four maze quadrants (the target quadrant). The platform remained in the same quadrant during the entire experiment. The training consisted of 4 trials per day for $4 \mathrm{~d}$ with each trial having a time limit of $60 \mathrm{~s}$ and with an interval between trials of approximately $60 \mathrm{~s}$. Each mouse had to swim until it climbed onto the submerged platform. After climbing onto the platform, the animal remained there for $30 \mathrm{~s}$ before the commencement of the next trial. If the mouse failed to reach the escape platform within the maximally allowed time of $60 \mathrm{~s}$, it was gently placed on the platform and allowed to remain there for $30 \mathrm{~s}$, and the time to reach the platform (latency) was recorded as $60 \mathrm{~s}$. On the fifth day, a spatial probe test was conducted. Each mouse was given one $60 \mathrm{~s}$ retention test trial in which the platform had been removed from the tank. The time spent in the target quadrant was recorded. The time spent in the target quadrant indicates the degree of memory consolidation that took place after learning.

\section{Protein, lipid peroxidation, glutathione and superoxide dismutase assays}

After completing the Morris water maze test, the animals were sacrificed and their brains were quickly removed to dissect the hippocampus and cerebral cortex. The dissected brains were homogenized in $0.1 \mathrm{~mol} / \mathrm{L}$ phosphate buffer (PB, pH 7.4). The homogenate was used to estimate the amount of lipid peroxidation, glutathione and superoxide dismutase. The amounts of protein, tissue lipid peroxidation, glutathione and superoxide dismutase were determined using kits (Nanjing Jiancheng Bioengineering Institute, Nanjing, China). The homogenate was centrifuged for $30 \mathrm{~min}$ at $3000 \times g$ at $4^{\circ} \mathrm{C}$, and the supernatant was used for enzyme assays. Glutathione (GSH) levels were determined using the DTNB-GSH reductase recycling method $^{[21]}$. The levels of malondialdehyde (MDA), an intermediate product of lipid peroxidation, were determined with the thiobarbituric acid (TBA) reaction ${ }^{[22]}$. The protein content was measured by the method of Bradford ${ }^{[23]}$ using bovine serum albumin as a standard.

\section{Histological analysis by hematoxylin-eosin (HE) staining}

The hippocampi of two mice from each group were chosen for hematoxylin-eosin (HE) staining. The mice were sacrificed and immediately transcardially perfused with $0.1 \mathrm{~mol} / \mathrm{L}$ phosphate buffer, $\mathrm{pH} 7.4$, followed by freshly prepared $4 \%$ paraformaldehyde in $0.1 \mathrm{~mol} / \mathrm{L}$ phosphate buffer. The brains were removed and fixed in $4 \%$ paraformaldehyde in $0.1 \mathrm{~mol} / \mathrm{L}$ phosphate buffer ( $\mathrm{pH} \mathrm{7.4)}$ at $4^{\circ} \mathrm{C}$ for more than $24 \mathrm{~h}$. Coronal blocks were embedded in paraffin for staining. The hippocampi stained with HE were analyzed under a microscope at $400 \times$ magnification.

\section{Statistical analysis}

The data were expressed as the mean \pm SD. The data were analyzed using a one-way factorial analysis of variance (ANOVA). Tukey's test was then performed to compare treated samples, and the differences were considered to be significant when $P<0.05$. 


\section{Results}

AA attenuates Glu-induced toxicity in SH-SY5Y cells

The treatment of SH-SY5Y cells with Glu (8 or $10 \mathrm{mmol} / \mathrm{L}$ ) for $24 \mathrm{~h}$ markedly reduced cell viability (Figure 1A). A concentration of $10 \mathrm{mmol} / \mathrm{L}$ Glu was chosen for our subsequent experiments. To examine the neuroprotective effects of AA, the cells were preincubated with different concentrations of AA (0.01$100 \mathrm{nmol} / \mathrm{L}$ ) for $24 \mathrm{~h}$, followed by exposure to $10 \mathrm{mmol} / \mathrm{L}$ Glu for $24 \mathrm{~h}$. AA provided protection against Glu-induced injury, and the strongest protective effect was achieved with $10 \mathrm{nmol} / \mathrm{L} \mathrm{AA}$ (Figure 1B).

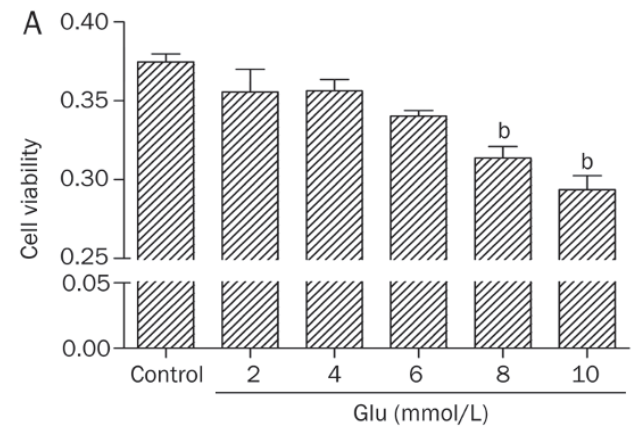

\section{Effects of AA on Glu-induced apoptosis}

Apoptosis was assessed using Hoechst staining and flow cytometry. As shown in Figure 2A, the exposure of cells to 10 $\mathrm{mmol} / \mathrm{L}$ Glu resulted in chromatin condensation but not DNA fragmentation. A similar form of chromatin condensation has recently been observed in HT22 cells and cerebellar granule neurons exposed to $\mathrm{Glu}^{[24,25]}$. Pretreatment with AA alleviated Glu-induced nuclear morphological alterations. The flow cytometry results (Figure 2C) demonstrated that stimulation with $10 \mathrm{mmol} / \mathrm{L} \mathrm{Glu}$ produced apoptosis in $7.38 \%$ of the cells compared with $1.28 \%$ of the control group. AA $10 \mathrm{nmol} / \mathrm{L}$ treatment reduced the incidence of apoptosis to $2.98 \%$.

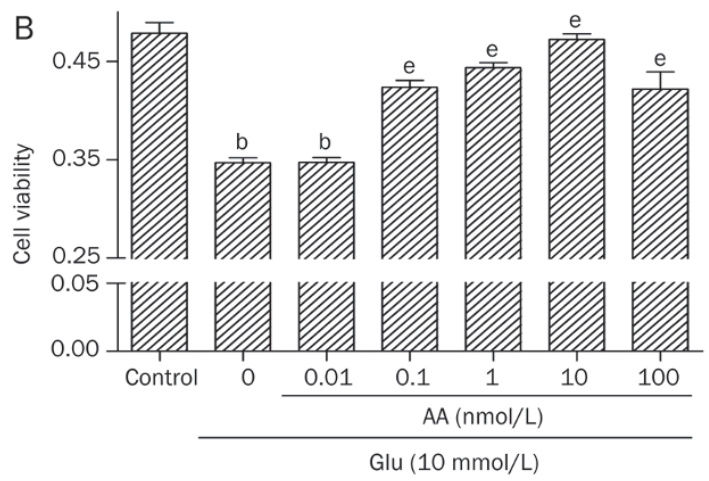

Figure 1. Inhibition by AA of Glu-induced cell death. (A) Cell viability was determined using MTT assay. Cells were exposed to 2-10 mmol/L Glu for $24 \mathrm{~h}$ without AA pretreatment. (B) Cells were preincubated with different concentrations of AA for $24 \mathrm{~h}$, and then exposed to $10 \mathrm{mmol} / \mathrm{L}$ Glu for $24 \mathrm{~h}$. ${ }^{\mathrm{b}} P<0.05$ vs control. ${ }^{\mathrm{e}} P<0.05$ vs Glu. All data are expressed as mean \pm SD of 5 replicate values in 3 separate experiments.

A

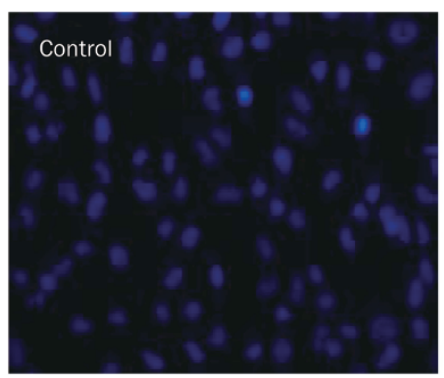

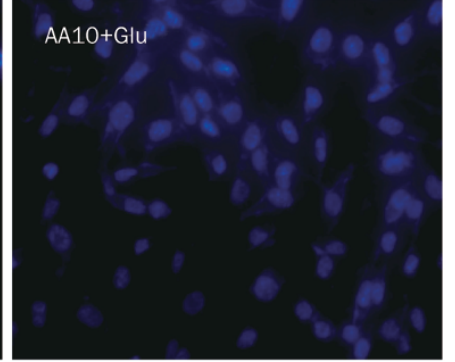

B
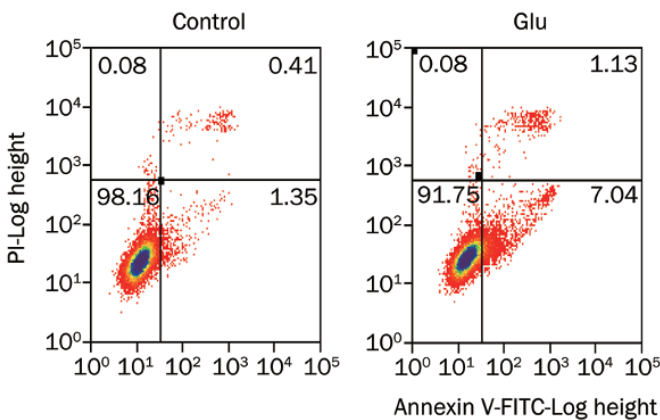

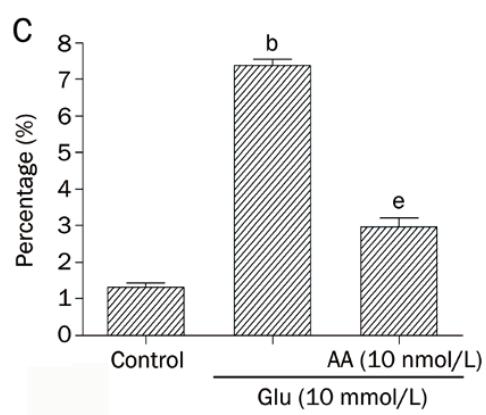

Figure 2. AA pretreatment significantly suppressed Glu-induced cell apoptosis. (A) AA pretreatment significantly decreased Glu-induced nuclear condensation. Apoptosis was determined morphologically by staining with Hoechst 33342 (original magnification 400×). AA10, 10 nmol/L AA. (B) Flow cytometric analyses with Annexin V-FITC and PI labels of cultured SH-SY5Y cells. (C) Percentage of apoptotic cells in total cells $(n=3)$. ${ }^{b} P<0.05$ vs control. ${ }^{e} P<0.05$ vs Glu. 
$A A$ reduces $R O S$ generation and restores the mitochondrial membrane potential (MMP) following Glu stimulation

Previous studies have demonstrated that stimulation by Glu can result in excessive ROS generation and a decrease in the mitochondrial membrane potential ${ }^{[26,27]}$. We sought to determine whether AA has the ability to modulate the mitochondrial membrane potential and levels of intracellular ROS following excitotoxic stimulation. As shown in Figure 3B, FACS analysis revealed that $10 \mathrm{mmol} / \mathrm{L}$ Glu treatment increased ROS levels compared with the control group, whereas 10 nmol/L AA pretreatment significantly reduced Glu-induced ROS generation. Representative fluorescence photomicrographs (Figure 3A) were consistent with the FACS results. To assess the effect of AA on the changes in the MMP induced by Glu, fluorescence spectrometry and fluorescence microscopy analyses were performed using JC-1 staining. Glu induced a decline in the MMP compared with the control. This decline in MMP was prevented by AA (Figures 3C, 3D). These results indicate that AA may prevent Glu-mediated neurotoxicity partially through a reduction in ROS production and a restoration of MMP.

\section{Effects of AA on the expression of PGC-1 $\alpha$ and Sirt1}

The silent information regulator 2 family of proteins (sirtuins) are NAD-dependent deacetylases that are believed to regulate survival and longevity ${ }^{[28]}$. Mammalian species have seven different sirtuin family members with the closest relative to the yeast sirtuin being Sirt1. Its beneficial role in neurodegenerative diseases has been studied extensively, and it holds great potential as a therapeutic target for neurodegeneration ${ }^{[29]}$. Peroxisome proliferator-activated receptor $\gamma$ coactivator a (PGC-1a) controls mitochondrial biogenesis and function ${ }^{[30]}$
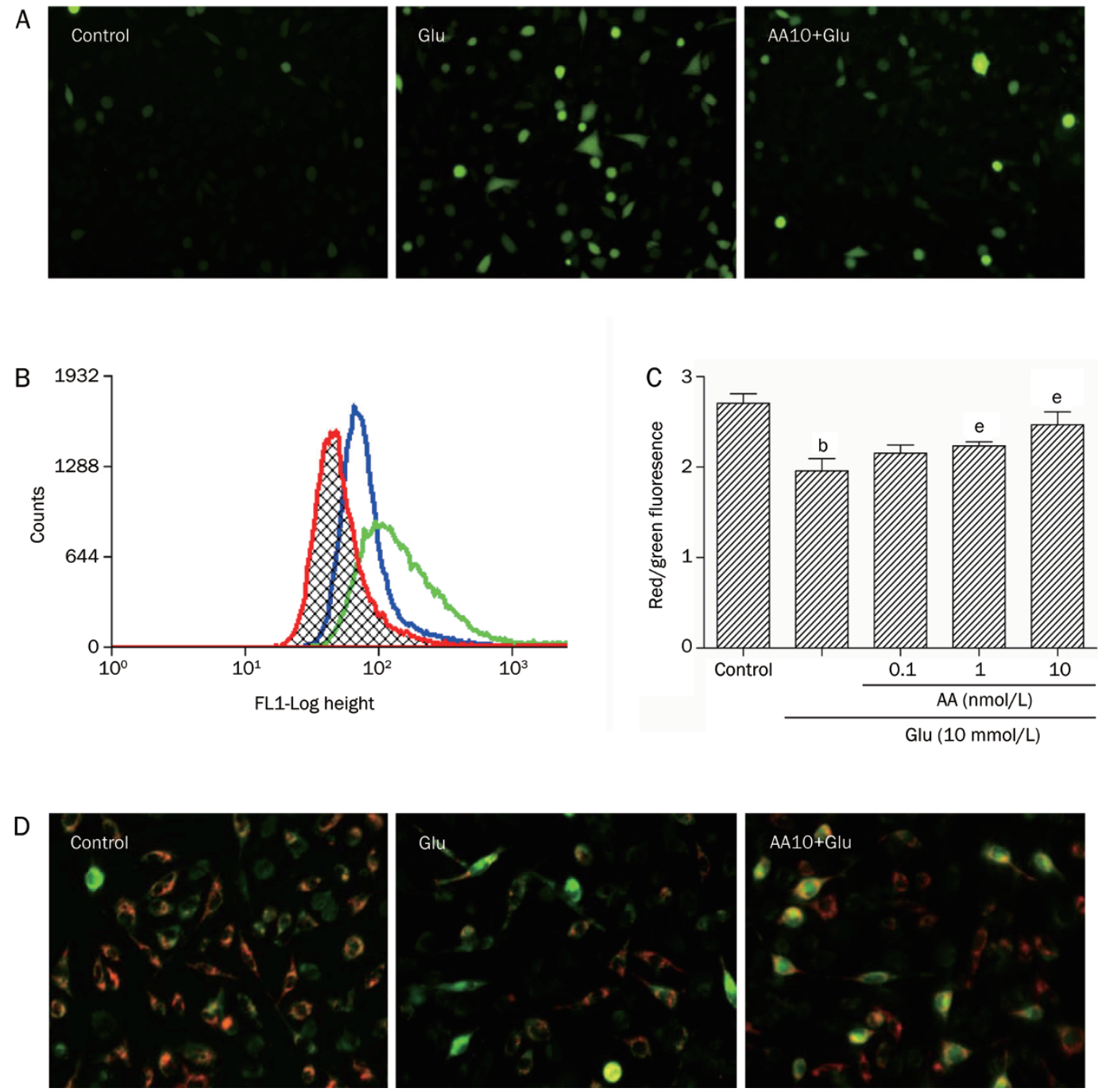

Figure 3. AA reduced ROS generation and restored MMP following Glu stimulus. (A) Cells were exposed to $10 \mathrm{mmol} / \mathrm{L}$ Glu with or without $10 \mathrm{nmol} / \mathrm{L}$ AA pretreatment. Representative fluorescence photographs (100x). AA10: $10 \mathrm{nmol} / \mathrm{L} \mathrm{AA}$. (B) Levels of ROS were analysed by FACS. Representative flow cytometry graph showing the DCF staining ( $n=3)$. Red peak: control; green peak: $10 \mathrm{mmol} / \mathrm{L} \mathrm{Glu;} \mathrm{blue} \mathrm{peak:} 10 \mathrm{nmol} / \mathrm{L} \mathrm{AA+10} \mathrm{mmol/L} \mathrm{Glu.} \mathrm{(C)} \mathrm{MMP} \mathrm{of}$ cells with $24 \mathrm{~h}$ Glu stimulus in the absence or presence of AA, determined by fluorescence spectrometry. (D) Representative fluorescence photographs of cellular mitochondrial membrane potential (400x). Mean \pm SD $(n=3)$ are shown. ${ }^{b} P<0.05$ vs control. ${ }^{e} P<0.05$ vs Glu. 

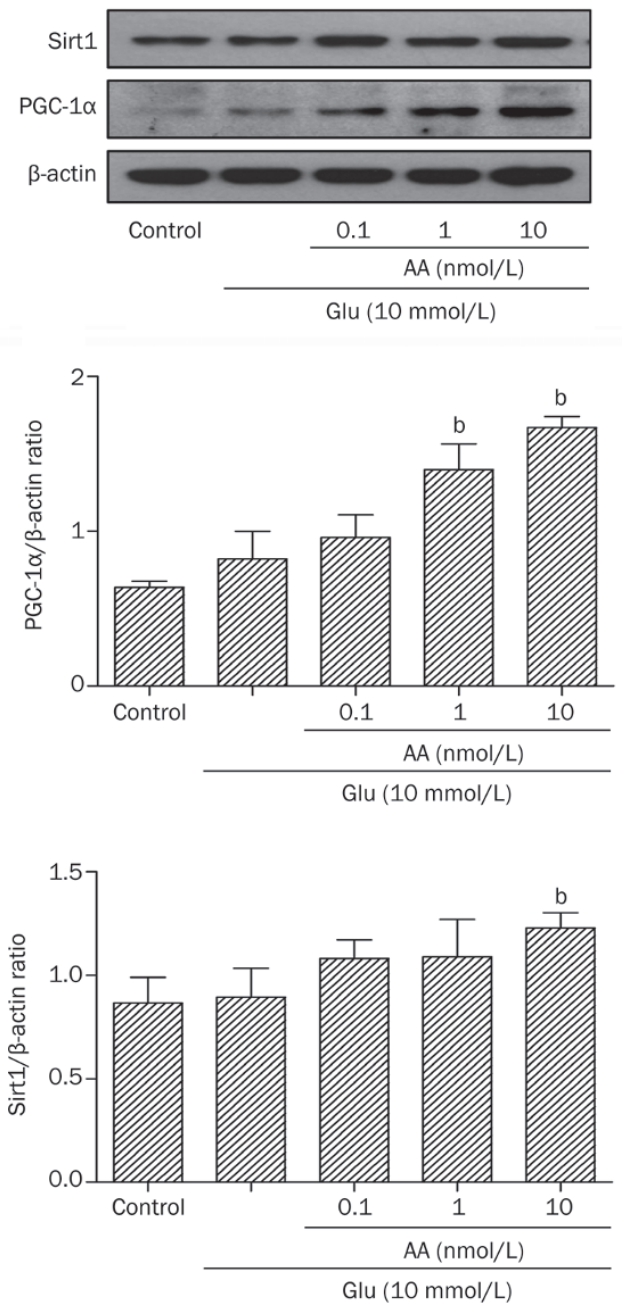

Figure 4. Effects of AA on Glu-stimulated expressions of Sirt1 and PGC-1 $\alpha$. Sirt1 and PGC-1 $\alpha$ expressions in cells pretreated with AA $(0.1-10$ nmol/L). Mean \pm SD. $n=3$. ${ }^{b} P<0.05$ vs Glu group.

and thereby plays an important role in brain energy homeostasis and neurodegenerative diseases ${ }^{[31,32]}$. Recently, an increasing number of studies have indicated that Sirt1 can interact with and regulate the activity of PGC- $1 \mathrm{a}^{[33,34]}$. Because of these findings, we examined whether AA affects the expression of Sirt1 or PGC-1a in Glu-treated cells. According to Western blot analysis (Figure 4), cells preincubated with AA showed an upregulation of Sirt1 and PGC-1a compared with the Gluonly group, and this upregulation may have prevented Gluinduced injury in these cells.

\section{Effects of AA on MSG-induced cognitive deficits}

The amount of time mice required to find the hidden platform during the acquisition phase of the water maze experiment is presented in Figure 5A. The mean latencies for all groups were similar on the first day; in the following days, controls rapidly improved to locate the hidden platform, whereas the MSG group tended to require more time than controls. The difference in latency to locate the platform between the MSG
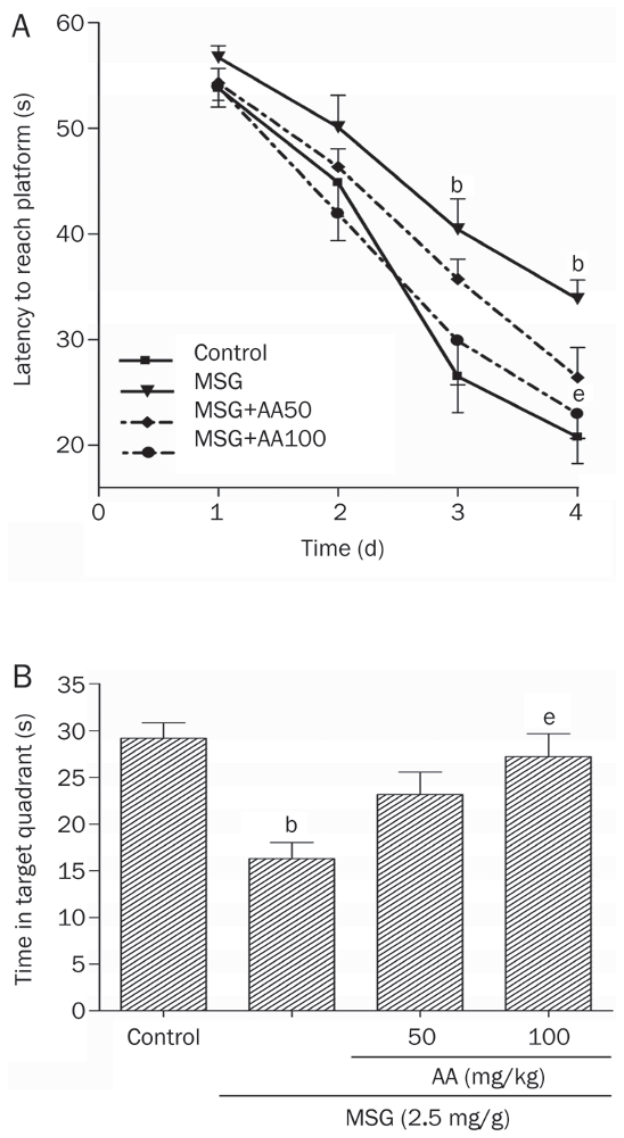

Figure 5. Effects of MSG and treatment with AA on the performance of spatial memory acquisition. (A) Latencies of mice during the $4 \mathrm{~d}$ test. (B) Times on $d 5$ that mice spent in the target quadrant with the platform removed. Mean \pm SEM. $n=10$. ${ }^{\mathrm{b}} P<0.05$ vs control. ${ }^{\mathrm{e}} P<0.05$ vs MSG.

and control groups was significant by $\mathrm{d} 3$ and 4 , whereas improvement of the AA-treated group versus the MSG group became significant on $\mathrm{d} 4$. The MSG-treated animals showed a reduced ability to find the platform, and this poorer performance was partially prevented by chronic treatment with AA. Animals administered the high dose of AA showed a better capacity to reach the platform than those administered the low dose. In the spatial probe on 5 of the trial, in which the platform was removed and mice were given one $60 \mathrm{~s}$ retention test trial, the MSG group spent less time in the platform quadrant than the control group (Figure 5B). In contrast, the mice treated with AA spent a significantly longer time in this quadrant than the MSG group. The $100 \mathrm{mg} / \mathrm{kg}$ dose was associated with better memory consolidation.

\section{Effects of AA on MSG-induced oxidative stress}

It has been suggested that MSG can induce oxidative stress in the rat brain and that antioxidants may be effective at ameliorating this effect ${ }^{[35]}$. Therefore, we investigated the levels of lipid peroxidation and the activity of an antioxidant enzyme in the hippocampus and cortex. The MSG-induced lipid peroxidation levels [determined using malondialdehyde (MDA)] 
in the hippocampus and cortex of mice increased significantly, whereas glutathione levels and SOD activity decreased. The treatment with AA restored the levels of lipid peroxidation and glutathione and the activity of SOD toward their control values (Table 1).

Table 1. MSG-induced changes in mouse brain oxidative stress parameters and their responses to administration of AA. Values are expressed as mean \pm SEM. $n=8$. ${ }^{\mathrm{b}} P<0.05$ vs control. ${ }^{\mathrm{e}} P<0.05$ vs MSG.

\begin{tabular}{lccc}
\hline & $\begin{array}{c}\text { GSH } \\
\text { (mg/g prot) }\end{array}$ & $\begin{array}{c}\text { SOD } \\
\text { (U/mg prot })\end{array}$ & $\begin{array}{c}\text { MDA } \\
(\mathrm{nmol} / \mathrm{mg} \text { prot })\end{array}$ \\
\hline Control & $7.22 \pm 0.32$ & $402 \pm 23$ & $8.44 \pm 1.44$ \\
MSG & $6.72 \pm 0.44$ & $365 \pm 34$ & $13.68 \pm 3.37^{\mathrm{b}}$ \\
MSG+AA $50 \mathrm{mg} / \mathrm{kg}$ & $7.71 \pm 0.45^{\mathrm{e}}$ & $372 \pm 24$ & $10.38 \pm 0.90^{\mathrm{e}}$ \\
MSG+AA $100 \mathrm{mg} / \mathrm{kg}$ & $7.90 \pm 0.51^{\mathrm{e}}$ & $381 \pm 20$ & $8.73 \pm 0.91^{\mathrm{e}}$ \\
\hline
\end{tabular}

\section{Effects of AA on MSG-induced injury of the hippocampus}

Neonatal MSG treatment produces degenerative changes in the developing brain; many studies have found that the injury is attributable to the destruction of the hippocampus ${ }^{[36]}$. In HE-stained sections, neuronal damage was manifested in the MSG group. The pyramidal layered structure disintegrated, and neuronal loss was found in the CA1 region. Neurons with pyknotic or shrunken nuclei were also observed in the CA3 region (Figure 6). These injuries were significantly attenuated by AA treatment.

\section{Discussion}

In the present study, we report that AA protected SH-SY5Y cells from Glu-induced injury in vitro and improved learning and memory deficits in the MSG-induced dementia animal model in vivo.

First, our data analysis shows that AA significantly protected cells from Glu excitotoxicity (Figure 1), and AA itself caused no conspicuous alterations in the growth of SH-SY5Y cells (data not shown). The neuroprotective effect of AA in the MTT assay paralleled the morphological analyses obtained with Hoechst 33342 staining and the flow cytometry assay. In addition, an important result demonstrated that 0.1-100 $\mathrm{nmol} / \mathrm{L}$ AA acted against Glu toxicity; however, with increasing concentrations of $\mathrm{AA}$, the protective ability decreased and even showed toxic effects (data not shown). This is because at high doses, AA can induce apoptosis via the activation of caspase- 9 and -3 and increased intracellular free $\mathrm{Ca}^{2+[37,38]}$.

The overactivation of glutamate receptors has been reported to induce an excessive influx of $\mathrm{Ca}^{2+}$, following depolarization of the mitochondrial membrane and increased production of $\operatorname{ROS}^{[26]}$. Mitochondria are known to generate ROS due to mitochondrial electron flow in the respiratory chain ${ }^{[39]}$. Meanwhile, mitochondria themselves are vulnerable to ROS, and excessive ROS can induce mitochondrial damage. This interaction between mitochondrial dysfunction and ROS genera-

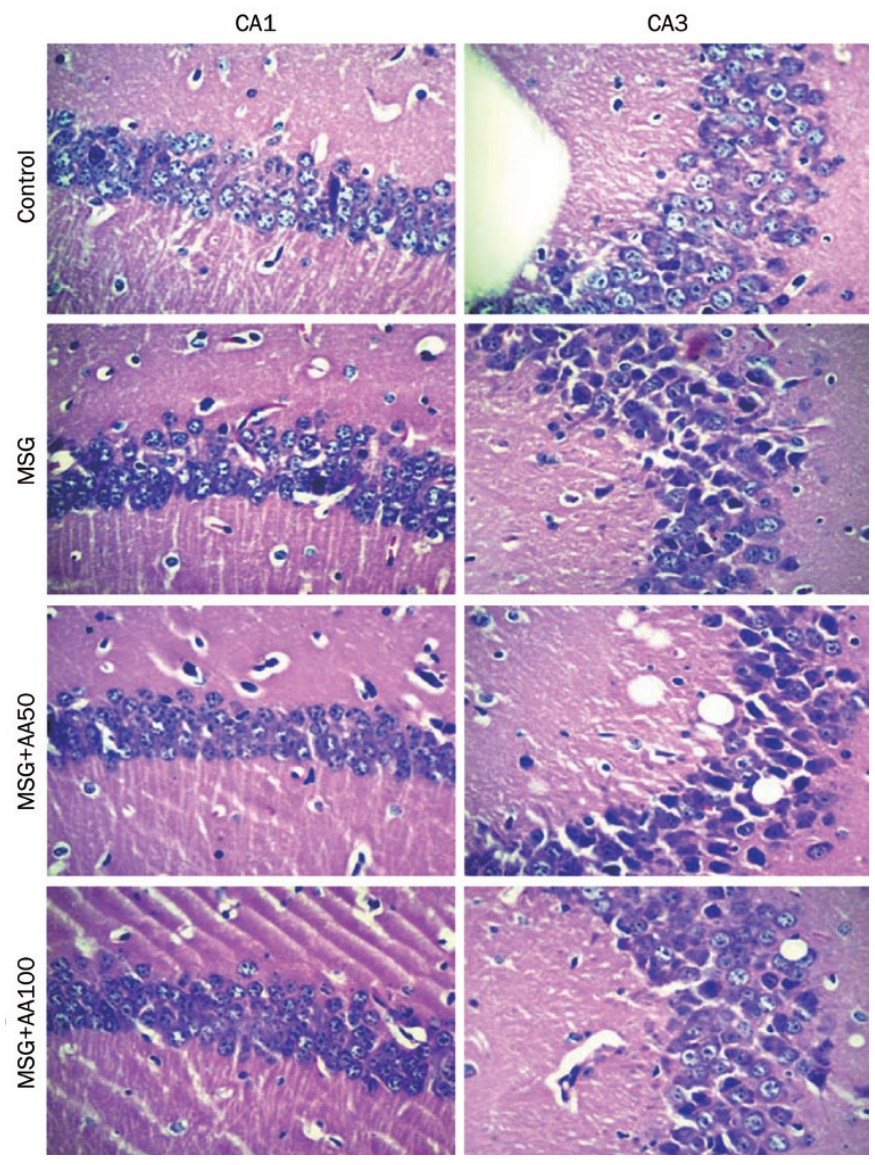

Figure 6. Effect of AA treatment on $\mathrm{CA} 1$ and $\mathrm{CA} 3$ neuron densities in hippocampus. Representative pictures obtained by HE staining (400x). When compared with the MSG-treated group, CA1 and CA3 neurons appear well preserved in AA-treated mice. MSG, 2.5 mg/g MSG; AA50, 50 mg/kg AA; AA100: 100 mg/kg AA.

tion may contribute to an understanding of why AA inhibits cell death during neurotoxicity. On the basis of the above discussion, reducing the intracellular ROS levels and restoring the MMP significantly affects the neuroprotective function of AA. Our data suggest that, at a concentration of $10 \mathrm{mmol} / \mathrm{L}$, Glu increases ROS levels. Pretreatment with AA reduced ROS levels in Glu-injured cells (Figure 3). These results are consistent with those of our previous study, which showed that AA has hydroxyl radical-scavenging activity in cell-free systems ${ }^{[40]}$. The disruption of the MMP may lead to cytochrome c release and activation of caspases that may lead to cell death. In our experiments, AA attenuated the decline of the MMP induced by Glu. Our data corroborate the results of a previous study, which reported that AA prevents the collapse of the MMP in rotenone-induced neuronal damage ${ }^{[14]}$ and in the oxygenglucose deprivation (OGD) cell culture model of ischemia ${ }^{[17]}$.

Recently, an increasing number of studies have indicated that the regulation of mitochondrial biogenesis may be beneficial for neuronal recovery and survival in neurodegenerative disorders ${ }^{[32,41,42]}$. PGC-1a has been shown to be a master regulator of mitochondrial biogenesis and cellular energy 
metabolism. PGC-1a increases the expression of a major antioxidant enzyme of mitochondria, superoxide dismutase 2 (SOD2) $^{[43]}$, and powerfully suppresses reactive oxygen species (ROS) in vivo ${ }^{[32]}$. Furthermore, PGC-1a knockout mice are much more sensitive to damage by oxidative stress, displaying apoptotic cell death in the dopaminergic cells of the substantia nigra and in hippocampal neurons ${ }^{[32]}$. From these findings, it seems reasonable that therapeutic agents that activate PGC-1a could successfully treat those neurodegenerative diseases in which mitochondrial dysfunction and oxidative damage play an important pathogenic role. Sirt1 - an NAD-dependent deacetylase that has been linked to longevity - interacts with and regulates the activity of PGC- $1 a^{[34,44]}$. It was reported that Sirt1 can deacetylate transcription factors such as p53 and the forkhead transcription factor (FOXO) family of proteins and thereby reduce p53, FOXO-induced apoptosis ${ }^{[45,46]}$. Thus, Sirt1 may be another agent capable of playing a therapeutic role in neurodegenerative disease ${ }^{[29]}$. Due to the considerable effects that PGC-1a and Sirt1 have on neuronal function, we examined if they mediate the neuroprotective effects of AA. A novel finding is that pretreatment with AA (0.1-10 nmol/L) prior to Glu stimulation dose-dependently increases the expression of PGC-1a and Sirt1 at the same time. This result is consistent with the finding that Sirt1 can regulate PGC-1a as mentioned above. Thus, it is possible that the upregulation of PGC-1a and Sirt1 is responsible for the neuroprotective effects of AA. Another neuroprotective agent, resveratrol, also activates the Sirt1 pathway ${ }^{[47,48]}$. Interestingly, we also found that after exposure to Glu alone, the cells show a slight increase in PGC-1a. This change in PGC-1a may reflect the cell's intrinsic response to stressful stimuli, but AA pretreatment largely increased the expression of PGC-1a compared with Glu stimulation alone. This is consistent with a previous report that NMDA can directly induce the expression of PGC-1a in neuronal cells ${ }^{[49]}$.

Furthermore, the neuroprotective function of AA ameliorates MSG-induced cognitive deficits in vivo. The neonatal administration of MSG causes cognitive deficits in adult animals $^{[50-52]}$. The Morris water maze is a commonly used method for evaluating learning and memory. MSG-induced deficits in learning and memory were revealed in the mice, and memory was enhanced by AA. The hippocampus has an important role in spatial learning and memory ${ }^{[53]}$. Damage to and morphological changes of the CA1 hippocampal structure has been described in MSG-treated animals ${ }^{[36,54]}$. Our data confirmed that the CA1 hippocampal structure underwent disintegration. We also found that the CA3 pyramidal neurons became pyknotic or shrunken, and this damage was significantly prevented by simultaneous administration of AA. However, the CA3 hippocampal neurons appear to be less damaged when analyzed in terms of tissue volume and cell numbers instead of morphology ${ }^{[54]}$. Previous studies have suggested that the oxidative stress induced by MSG might contribute to hippocampal impairment ${ }^{[51,55]}$. AA is viewed as a promising antioxidant candidate; it sufficiently abated oxidative stress in MSG-treated rodents by enhancing both GSH levels and SOD activity and by reducing MDA levels. Therefore, it is hypothesized that the attenuation of oxidative stress, following induced hippocampal damage, is responsible for the effects of AA against MSG-induced dementia.

In conclusion, our data support the notion that AA attenuates cognitive deficits in an animal model of MSG-induced dementia. In addition, AA offered beneficial effects in Gluinduced cellular injury by suppressing oxidative stress and protecting mitochondria. Taking these in vitro and in vivo results together, especially the protection of mitochondria by AA, AA and related compounds may develop into a new therapeutic approach for preventing and/or treating neuronal damage and degenerative disorders.

\section{Acknowledgements}

We thank Dr Edward SHARMAN, University of California, Irvine, for his careful reading and editing of this manuscript. This work was supported by the Excellent Young Teachers Program of MOE and grants from Jiangsu University (07JDG012), Jiangsu Natural Sciences Foundation (2008249), and the National Natural Science Foundation of China (31171143).

\section{Author contribution}

Min-fang XU and Jing GAO designed the experiments; Minfang XU and Yu-yun XIONG performed the experiments; Minfang XU analyzed the data and drafted the manuscript; Jiankang LIU revised the manuscript and offered valuable advice about all of the experiments; Jin-jun QIAN and Li ZHU offered helpful suggestions and discussion; and Jing GAO revised the paper and supervised the experiments.

\section{References}

1 Hague SM, Klaffke S, Bandmann O. Neurodegenerative disorders: Parkinson's disease and Huntington's disease. J Neurol Neurosur Ps 2005; 76: 1058-63.

2 Dumont RJ, Okonkwo Do, Verma S, Hurlbert RJ, Boulos PT, Ellegala DB, et al. Acute spinal cord injury, part I: pathophysiologic mechanisms. Clin Neuropharmacol 2001; 24: 254-64.

3 Lankiewicz S, Marc Luetjens C, Truc Bui N, Krohn AJ, Poppe M, Cole $\mathrm{GM}$, et al. Activation of calpain I converts excitotoxic neuron death into a caspase-independent cell death. J Biol Chem 2000; 275: 17064-71.

4 Nicholls DG. Mitochondrial calcium function and dysfunction in the central nervous system. BBA-Bioenergetics 2009; 1787: 1416-24.

5 Pivovarova NB, Andrews SB. Calcium-dependent mitochondrial function and dysfunction in neurons. FEBS J 2010; 277: 3622-36.

6 Lazzarino G, Vagnozzi R, Tavazzi B, Pastore FS, Di Pierro D, Siragusa $\mathrm{P}$, et al. MDA, oxypurines, and nucleosides relate to reperfusion in short-term incomplete cerebral ischemia in the rat. Free Radic Biol Med 1992; 13: 489-98.

7 Greenamyre JT, Penney JB, Young AB, D'Amato CJ, Hicks SP, Shoulson I. Alterations in L-glutamate binding in Alzheimer's and Huntington's diseases. Science 1985; 227: 1496-9.

8 Armstrong JS. Mitochondrial medicine: pharmacological targeting of mitochondria in disease. Br J Pharmacol 2007; 151: 1154-65.

9 Liu JK, Ames BN. Reducing mitochondrial decay with mitochondrial nutrients to delay and treat cognitive dysfunction, Alzheimer's 
disease, and Parkinson's disease. Nutr Neurosci 2005; 8: 67-89.

10 Kumar MHV, Gupta YK. Effect of different extracts of Centella asiatica on cognition and markers of oxidative stress in rats. J Ethnopharmacol 2002; 79: 253-60.

11 Wattanathorn J, Mator L, Muchimapura S, Tongun T, Pasuriwong O, Piyawatkul N, et al. Positive modulation of cognition and mood in the healthy elderly volunteer following the administration of Centella asiatica. J Ethnopharmacol 2008; 116: 325-32.

12 Gnanapragasam A, Yogeeta S, Subhashini R, Ebenezar KK, Sathish $\mathrm{V}$, Devaki T. Adriamycin induced myocardial failure in rats: protective role of Centella asiatica. Mol Cell Biochem 2007; 294: 55-63.

13 Soumyanath A, Zhong YP, Gold SA, Yu XL, Koop DR, Bourdette D, et al. Centella asiatica accelerates nerve regeneration upon oral administration and contains multiple active fractions increasing neurite elongation in-vitro. J Pharm Pharmacol 2005; 57: 1221-29.

14 Xiong YY, Ding HQ, Xu MF, Gao J. Protective effects of asiatic acid on rotenone- or $\mathrm{H}_{2} \mathrm{O}_{2}$-induced injury in SH-SY5Y cells. Neurochem Res 2009; 34: 746-54.

15 Lee MK, Kim SR, Sung SH, Lim DY, Kim H, Choi H, et al. Asiatic acid derivatives protect cultured cortical neurons from glutamate-induced excitotoxicity. Res Commun Mol Pathol Pharmacol 2000; 108: 7586.

16 Jew SS, Yoo CH, Lim DY, Kim H, Mook-Jung I, Jung MW, et al. Structure-activity relationship study of asiatic acid derivatives against beta amyloid (A beta)-induced neurotoxicity. Bioorg Med Chem Lett 2000; 10: 119-21.

17 Krishnamurthy RG, Senut MC, Zemke D, Min JY, Frenkel MB, Greenberg EJ, et al. Asiatic acid, a pentacyclic triterpene from Centella asiatica, is neuroprotective in a mouse model of focal cerebral Ischemia. J Neurosci Res 2009; 87: 2541-50.

18 Ishikawa K, Kubo T, Shibanoki S, Matsumoto A, Hata H, Asai S. Hippocampal degeneration inducing impairment of learning in rats: model of dementia? Behav Brain Res 1997; 83: 39-44.

19 Urena-Guerrero ME, Lopez-Perez SJ, Beas-Zarate C. Neonatal monosodium glutamate treatment modifies glutamic acid decarboxylase activity during rat brain postnatal development. Neurochem Int 2003; 42: 269-76.

20 Morris RG, Garrud P, Rawlins JN, O'Keefe J. Place navigation impaired in rats with hippocampal lesions. Nature 1982; 297: 681-3.

21 Anderson ME. Determination of glutathione and glutathione disulfide in biological samples. Methods Enzymol 1985; 113: 548-55.

22 Tzeng WF, Lee JL, Chiou TJ. The role of lipid peroxidation in menadione-mediated toxicity in cardiomyocytes. J Mol Cell Cardiol 1995; 27: 1999-2008.

23 Bradford MM. A rapid and sensitive method for the quantitation of microgram quantities of protein utilizing the principle of protein-dye binding. Anal Biochem 1976; 72: 248-54.

24 Elphick LM, Hawat M, Toms NJ, Meinander A, Mikhailov A, Eriksson $\mathrm{JE}$, et al. Opposing roles for caspase and calpain death proteases in L-glutamate-induced oxidative neurotoxicity. Toxicol Appl Pharm 2008; 232: 258-67.

25 Bezvenyuk Z, Miettinen R, Solovyan V. Chromatin condensation during glutamate-induced excitotoxicity of celebellar granule neurons precedes disintegration of nuclear DNA into high molecular weight DNA fragments. Mol Brain Res 2003; 110: 140-6.

26 Atlante A, Calissano P, Bobba A, Giannattasio S, Marra E, Passarella S. Glutamate neurotoxicity, oxidative stress and mitochondria. FEBS Lett 2001; 497: 1-5.

27 Ankarcrona M, Dypbukt JM, Bonfoco E, Zhivotovsky B, Orrenius S, Lipton SA, et al. Glutamate-induced neuronal death: a succession of necrosis or apoptosis depending on mitochondrial function. Neuron
1995; 15: 961-73.

28 Grubisha 0, Smith BC, Denu JM. Small molecule regulation of Sir2 protein deacetylases. FEBS J 2005; 272: 4607-16.

29 Outeiro TF, Marques O, Kazantsev A. Therapeutic role of sirtuins in neurodegenerative disease. Biochim Biophys Acta 2008; 1782: 363-9.

30 Finck BN, Kelly DP. PGC-1 coactivators: inducible regulators of energy metabolism in health and disease. J Clin Invest 2006; 116: 615-22.

31 Cui LB, Jeong H, Borovecki F, Parkhurst CN, Tanese N, Krainc D. Transcriptional repression of PGC-alpha by mutant huntingtin leads to mitochondrial dysfunction and neurodegeneration. Cell 2006; 127: 59-69.

32 St-Pierre J, Drori S, Uldry M, Silvaggi JM, Rhee J, Jager S, et al. Suppression of reactive oxygen species and neurodegeneration by the PGC-1 transcriptional coactivators. Cell 2006; 127: 397-408.

33 Lagouge M, Argmann C, Gerhart-Hines Z, Meziane H, Lerin C, Daussin $\mathrm{F}$, et al. Resveratrol improves mitochondrial function and protects against metabolic disease by activating SIRT1 and PGC-1 alpha. Cell 2006; 127: 1109-22.

34 Nemoto S, Fergusson MM, Finkel T. SIRT1 functionally interacts with the metabolic regulator and transcriptional coactivator PGC-1 alpha. J Bio Chem 2005; 280: 16456-60.

35 Farombi EO, Onyema OO. Monosodium glutamate-induced oxidative damage and genotoxicity in the rat: modulatory role of vitamin C, vitamin E and quercetin. Hum Exp Toxicol 2006; 25: 251-9.

36 Beas-Zarate C, Perez-Vega MI, Gonzalez-Burgos I. Neonatal exposure to monosodium L-glutamate induces loss of neurons and cytoarchitectural alterations in hippocampal CA1 pyramidal neurons of adult rats. Brain Res 2002; 952: 275-81.

37 Cho CW, Choi DS, Cardone MH, Kim CW, Sinskey AJ, Rha C. Glioblastoma cell death induced by asiatic acid. Cell Biol Toxicol 2006; 22 : 393-408.

38 Park BC, Bosire KO, Lee ES, Lee YS, Kim JA. Asiatic acid induces apoptosis in SK-MEL-2 human melanoma cells. Cancer Lett 2005; 218: 81-90.

39 Nicholls DG. Mitochondrial dysfunction and glutamate excitotoxicity studied in primary neuronal cultures. Curr Mol Med 2004; 4: 149-77.

40 Gao J, Tang XH, Dou H, Fan YM, Zhao XN, Xu Q. Hepatoprotective activity of terminalia catappa $L$ leaves and its two triterpenoids. J Pharm Pharmacol 2004; 56: 1449-55.

41 Clark J, Simon DK. Transcribe to survive: transcriptional control of antioxidant defense programs for neuroprotection in Parkinson's disease. Antioxid Redox Signal 2009; 11: 509-28.

42 Thomas B, Beal MF. Mitochondrial therapies for Parkinson's disease. Movement Disord 2010; 25: S155-S60.

43 Valle I, Alvarez-Barrientos A, Arza E, Lamas S, Monsalve M. PGC1alpha regulates the mitochondrial antioxidant defense system in vascular endothelial cells. Cardiovasc Res 2005; 66: 562-73.

44 Rodgers JT, Lerin C, Haas W, Gygi SP, Spiegelman BM, Puigserver P. Nutrient control of glucose homeostasis through a complex of PGC-1 alpha and SIRT1. Nature 2005; 434: 113-8.

45 Luo J, Nikolaev AY, Imai S, Chen D, Su F, Shiloh A, et al. Negative control of p53 by Sir2alpha promotes cell survival under stress. Cell 2001; 107: 137-48.

46 Giannakou ME, Partridge L. The interaction between FOXO and SIRT1: tipping the balance towards survival. Trends Cell Biol 2004; 14: 40812.

47 Pallas M, Casadesus G, Smith MA, Coto-Montes A, Pelegri C, Vilaplana $\mathrm{J}$, et al. Resveratrol and neurodegenerative diseases: activation of SIRT1 as the potential pathway towards neuroprotection. Curr Neurovasc Res 2009; 6: 70-81. 
48 Tan L, Yu JT, Guan HS. Resveratrol exerts pharmacological preconditioning by activating PGC-1 alpha. Med Hypotheses 2008; 71: 664-7.

49 Luo Y, Zhu WJ, Jia J, Zhang CY, Xu Y. NMDA receptor dependent PGC1 alpha up-regulation protects the cortical neuron against oxygenglucose deprivation/reperfusion injury. J Mol Neurosci 2009; 39: 262-8.

50 Olvera-Cortes E, Lopez-Vazquez MA, Beas-Zarate C, Gonzalez-Burgos I. Neonatal exposure to monosodium glutamate disrupts place learning ability in adult rats. Pharmacol Biochem Behav 2005; 82: 247-51.

51 Narayanan SN, Kumar RS, Paval J, Nayak S. Effect of ascorbic acid on the monosodium glutamate-induced neurobehavioral changes in periadolescent rats. Bratisl Lek Listy 2010; 111: 247-52.

52 Kiss P, Hauser D, Tamas A, Lubics A, Racz B, Horvath Z, et al.
Changes in open-field activity and novelty-seeking behavior in periadolescent rats neonatally treated with monosodium glutamate. Neurotox Res 2007; 12: 85-93.

53 Compton DM. Behavior strategy learning in rat: effects of lesions of the dorsal striatum or dorsal hippocampus. Behav Processes 2004; 67: 335-42.

54 Gonzalez-Burgos I, Velazquez-Zamora DA, Beas-Zarate C. Damage and plasticity in adult rat hippocampal trisynaptic circuit neurons after neonatal exposure to glutamate excitotoxicity. Int J Dev Neurosci 2009; 27: 741-5.

55 Thomas M, Sujatha KS, George S. Protective effect of piper longum linn on monosodium glutamate induced oxidative stress in rats. Indian J Exp Biol 2009; 47: 186-92. 